Instituto Internacional de Investigación y Desarrollo Tecnológico Educativo INDTEC, C.A.

DOI: https://doi.org/10.29394/Scientific.issn.2542-2987.2021.6.20.15.275-295

OAI-PMH: http://www.indteca.com/ojs/index.php/Revista Scientific/oai

Ensayo Original / Original Essay

\title{
El transitar en la investigación cualitativa: un acercamiento a la triangulación
}

\author{
Autora: Angela Esther Torres Ruiz \\ Universidad Nacional Experimental del Táchira, UNET \\ angtruiz@hotmail.com \\ San Cristóbal, Venezuela \\ https://orcid.org/0000-0003-2926-7174
}

Resumen

El presente escrito tiene como finalidad destacar la importancia de la triangulación al interior del ámbito investigativo, partiendo de la realización de un breve recorrido para realzar la trascendencia de la investigación cualitativa y el papel esencial que se asume en ella como determinante de la validez, sin adentrarse en planos explicativos profusos dada la inconmensurable riqueza teórica disponible sobre el tema. Para ello se han considerado los aportes de Vasilachis (2012); Denzin y Lincoln (2012), respecto a la esencia del abordaje cualitativo, así como las contribuciones teóricas de Alzás y Casa (2017); Ruiz (2012); Fernández y Postigo-Fuentes (2020), para consolidar el marco comprensivo sobre el papel de la triangulación. Acentuándose que aun cuando no de modo privativo, en el campo de las ciencias sociales, el abordaje cualitativo brinda la oportunidad de apreciar las singularidades de los fenómenos a partir de disímiles miradas que sumergen al investigador en un campo interpretativo fecundo. Subrayándose además, que para eludir los sesgos y las distorsiones de las interpretaciones, producto de las posturas filosóficas del investigador y las subjetividades que lo definen, la triangulación emerge como una herramienta substancial que procura asegurar el carácter acendrado de las reconstrucciones interpretativas de la realidad.

Palabras clave: investigación; triangulación; investigación cualitativa. Código de clasificación internacional: 5802.07 - Formación profesional.

Cómo citar este ensayo:

Torres, A. (2021). El transitar en la investigación cualitativa: un acercamiento a la triangulación. Revista Scientific, 6(20), 275-295, e-ISSN: 2542-2987. Recuperado de: https://doi.org/10.29394/Scientific.issn.2542-2987.2021.6.20.15.275-295

Fecha de Recepción: 05-01-2021
Fecha de Aceptación: 26-03-2021
Fecha de Publicación: 05-05-2021 
Instituto Internacional de Investigación y Desarrollo Tecnológico Educativo INDTEC, C.A.

DOI: https://doi.org/10.29394/Scientific.issn.2542-2987.2021.6.20.15.275-295

OAI-PMH: http://www.indteca.com/ojs/index.php/Revista Scientific/oai

Ensayo Original / Original Essay

\title{
The transition in qualitative research: an approach to triangulation
}

\begin{abstract}
The purpose of this document is to highlight the importance of triangulation within the research field, starting with a brief tour to highlight the significance of qualitative research and the essential role that is assumed in it as a determinant of validity, without delve into profuse explanatory plans given the immeasurable theoretical wealth available on the subject. For this, the contributions of Vasilachis (2012); Denzin and Lincoln (2012), regarding the essence of the qualitative approach, as well as the theoretical contributions of Alzás and Casa (2017); Ruiz (2012); Fernández and Postigo-Fuentes (2020), to consolidate the comprehensive framework on the role of triangulation. Emphasizing that even though not exclusively, in the field of social sciences, the qualitative approach offers the opportunity to appreciate the singularities of the phenomena from dissimilar views that immerse the researcher in a fertile interpretive field. Also underlining that to avoid biases and distortions of interpretations, product of the philosophical positions of the researcher and the subjectivities that define it, triangulation emerges as a substantial tool that seeks to ensure the correct character of the interpretative reconstructions of reality.
\end{abstract}

Keywords: investigation; triangulation; qualitative research. International classification code: 5802.07 - Professional training.

How to cite this essay:

Torres, A. (2021). The transition in qualitative research: an approach to triangulation. Revista Scientific, 6(20), 275-295, e-ISSN: 2542-2987. Recovered from: https://doi.org/10.29394/Scientific.issn.2542-2987.2021.6.20.15.275-295

Date Received: 05-01-2021
Date Acceptance: 26-03-2021
Date Publication: 05-05-2021 


\section{Introducción}

El carácter dinámico de la evolución del conocimiento, que identifica a los tiempos actuales, se fusiona con los complejos rasgos que definen la sociedad y, por ende, los fenómenos que en ella se suscitan. Es por ello que los investigadores, y muy especialmente los docentes, deben dotarse de herramientas conceptuales, técnicas y metodológicas que les permitan realizar acercamientos puntuales a las realidades sobre las cuales centran su atención, permitiéndose ampliar el espectro de posibilidades de exploración y afinar sus sentidos a fin de captar con el mayor detalle las singularidades que las componen.

Sin duda, el escenario incierto y ambiguo en el que se desarrollan las problemáticas socioeducativas conduce, de manera indefectible, a revisar de modo acucioso el soporte teórico que le permita al docente ahondar en la comprensión de esas realidades; esto emerge con un carácter imperioso, pues el campo investigativo en educación requiere de un marco interpretativo que conduzca según Rodríguez (2016): "[...] hacia el análisis de factores que han sido invisibilizados y permanecen ausentes en lo mostrado" (pág. 61). De allí que Tua (2021): acentúa la necesidad de que el docente desarrolle sus competencias investigativas y potencie sus habilidades a fin de "[...] que le permitan aplicar los conocimientos, para abordar de manera crítica la realidad [...]" (pág. 27).

Este reto que se plantea al docente le conduce de manera insoslayable, en los términos expresados por Flores, Meléndez y Mendoza (2019): a "[...] redimensionar los saberes, y reorientar esfuerzos [...]" (pág. 64); pues a juicio de los autores mencionados su accionar investigativo debe desarrollarlo bajo "[...] la firme convicción de que la ciencia debe estar conectada a la vida social" (pág. 64). Este lazo investigación-docencia debe sustentarse en un compendio teórico sólido que le permita adentrarse en cada escenario con una multiplicidad de herramientas interdisciplinares, entre las cuales ocupan lugar 
relevante las de orden metodológico, para aprehender las cualidades que definen cada realidad. En sintonía con lo anterior, Delgado y Alfonzo (2019), sostienen que es preciso:

[...] Reflexionar permanentemente, asumir una actitud crítica sobre lo aprendido en su formación y notificar saberes con sus pares y estudiantes, para establecer sinergias entre lo que se aprende e investiga, además sobre lo que se enseña y el para qué (págs. 203-204).

Así, para articular eficazmente la praxis con la investigación el docente debe asumir el rol de sujeto reflexivo, insertándose con certeza en su entorno para procurar realizar indagaciones que le conduzcan a plantear soluciones a las problemáticas que son competencia en su ámbito de acción. Debe acotarse, sin embargo, que el estrecho nexo entre ciencia, método científico y cuantificación se encuentra tan arraigado que es difícil advertir su influjo sobre el desarrollo de la praxis y la propensión a concederle mayor credibilidad; por lo que puede dejarse de lado la posibilidad de realizar pesquisas con una pluralidad de miradas complementarias que requieran un mayor grado de sensibilidad.

Esta preeminencia de las investigaciones con enfoque cuantitativo se aprecia de forma análoga en otros campos, como el relacionado con la investigación médica. En este sentido, Varela y Vives (2016): sostienen que en esa área los estudios cuantitativos "[...] son ampliamente aceptados por la comunidad científica, en tanto que la investigación de tipo cualitativo enfrenta el reto de defender la credibilidad y el rigor de sus métodos" (pág. 191). En contraste con lo anterior, Ugalde y Balbastre (2013): afirman que en el área de las ciencias sociales "los estudios cualitativos se prefieren por sus propiedades explicativas y su poder exploratorio" (pág. 181); insistiendo los autores precitados en que las visiones unificadas no son aplicables al hecho social, por lo que el enfoque cualitativo brinda la oportunidad de comprender de 
manera más profunda la realidad, dado que en ella se movilizan "[...] sentimientos, pensamientos e historias de los actores sociales que son captados a través de sus testimonios" (pág. 182).

Con lo anterior se quiere significar que la complejidad natural del hecho educativo genera choques entre la epistemología y las ontologías volátiles que subyacen en el acontecer áulico, por lo que se requiere romper los desacuerdos epistemológicos que subyacen en la dicotomía cuantitativocualitativo para buscar desde cada óptica una interpretación diáfana de la realidad. Ahora bien, posicionarse en la arista particular del enfoque cualitativo implica aprovechar su potencial como campo emergente de saberes que permite construir el conocimiento a partir de la experiencia, valorando, como lo deja saber Vasilachis (2012a): la diversidad, los matices y lo inédito.

Se debe resaltar que sumergirse en el tejido interactivo e interrelacionado entre el objeto de estudio y el contexto, cuando se indaga la realidad educativa, supone asumir el desafío de aplicar criterios de calidad y rigor que garanticen la robustez de los estudios; esta tarea es altamente compleja pues, las perspectivas teóricas y metodológicas que se manejan al interior del enfoque cualitativo son amplias y muestran una gran variabilidad.

Adicionándose a ello, el carácter interpretativo y constructivista que desde prismas hermenéuticos diversos otorgan significado a las realidades sociales; por ello, se requiere manejar las precisiones teóricas que garanticen el rigor metodológico y la validez de los hallazgos, punto en el cual la triangulación pasa a ocupar un sitial relevante. Justamente, para ampliar el horizonte comprensivo en lo relativo al carácter substancial del enfoque cualitativo en los estudios inherentes a las ciencias sociales y el papel primordial que representa en el proceso investigativo la triangulación se esboza en el subsiguiente discurrir algunos planteamientos y argumentos en torno a estos temas centrales en el campo de la investigación educativa.

En atención a lo expuesto, el objetivo central del presente escrito es 
subrayar la relevancia de la triangulación como herramienta que procura superar los sesgos interpretativos y le otorga mayor coherencia al proceso investigativo al posibilitar análisis más plurales y completos.

\section{Desarrollo}

De manera connatural las ciencias humanas por su carácter complejo requieren de posicionamientos de análisis que superan las potencialidades propias de las herramientas del paradigma científico tradicional, el cual se muestra incapaz de proveer explicaciones apropiadas y absolutamente admisibles de la realidad que nos envuelve y de la cual requerimos interpretaciones diáfanas a partir de lo que percibimos. Justamente, para evitar las concepciones atomizadas y exégesis fragmentadas de los fenómenos sociales surgió la investigación cualitativa, como forma de resignificar las vivencias y sentires de los actores expuestos a tensiones particulares en el mundo de facetas laberínticas en el cual les ha correspondido desplegar sus acciones.

Vista de este modo, la investigación cualitativa asume el desafío de capturar la experiencia vivida y recrearla en un texto reflexivo que lleve a construir intelecciones sobre aspectos crípticos de una realidad ajena y polimórfica. Así, tal investigación se concibe, en los términos propuestos por Denzin y Lincoln (2012a): como un campo de indagación por derecho propio, el cual "entrecruza disciplinas, áreas y objetos de estudio" (pág. 46); por lo que sirve de puente entre las visiones tradicionales rigurosas y las nuevas perspectivas interpretativas derivadas de las exigencias impuestas por el abordaje de fenómenos con rasgos contextuales, culturales e históricos particulares, cuya valoración supone la asunción de un camino intrincado.

En lo esencial, la investigación cualitativa es un campo interdisciplinario que determina un espacio donde las posiciones individuales y la acción logran entrecruzarse con el entramado que determina la cultura y los lazos sociales. 
De manera que, en la búsqueda del conocimiento interpretativo de la experiencia humana se convierte en un conjunto de prácticas multiparadigmáticas que, de acorde con lo establecido por Denzin y Lincoln (2012b): "nuclea diferentes comunidades interpretativas" (pág. 27). De este modo, ese proceso hermenéutico inherente a la perspectiva cualitativa en la investigación permite fusionar diversos prismas para consolidar la construcción social del conocimiento, a partir del íntimo lazo establecido entre el investigador y lo que estudia, sin dejar de valorar las constricciones del contexto que restringen la indagación.

Por lo que, en el transcurso de la creación de la imagen de la realidad, la investigación cualitativa, está representada para Denzin y Lincoln (2012c): como lo sostienen los autores precitados "ofrece un camino de doble circulación, entre lo público y lo privado, entre lo científico y lo sagrado, entre la investigación rigurosa y la expresión artística" (pág. 27).

Dentro de este marco, se infiere que el abordaje cualitativo enriquece las apreciaciones de los fenómenos al ofrecer una diversidad de posibles miradas sobre el mismo, dejando cada pesquisa la oportunidad de captar trazas singulares y esenciales que definen los mismos. En efecto, la contemplación cualitativa de los fenómenos ofrece renovadas y disímiles perspectivas, requiriendo que el investigador aprecie el escenario y a los actores en una perspectiva holística.

Debe resaltarse que el carácter creativo e interpretativo de la posición ideográfica del investigador cualitativo no le resta seriedad a este tipo de estudio, pues, como lo plantea Álvarez-Gayou (2003a): "Constituye una investigación sistemática conducida con procedimientos rigurosos, aunque no necesariamente homogéneos" (pág. 27). Lo anterior le permite a Vasilachis (2012b): destacar las bondades de la investigación cualitativa como herramienta que conduce a formas alternas de conocer y producir conocimiento, venciendo las tensiones entre la legitimación y la creación. 
Desde la perspectiva de la referida autora, su introducción abrió paso a nuevas corrientes de pensamiento que sobrepasaron los límites del conocimiento convencional, para revisar con nuevos prismas las prácticas con las que el individuo transforma su mundo y el mundo. Atendiendo a Vasilachis (2012c): es precisamente "el reconocimiento de la copresencia de una multiplicidad de formas de conocimiento" (pág. 12); el elemento que impulsa al investigador a acercarse a los diferentes fenómenos con una mayor sensibilidad.

Por otra parte, conviene destacar que las ciencias sociales y políticas, junto con las humanidades, tienen puntos de convergencia al compartir un abordaje interpretativo y aceptar formulaciones paradigmáticas múltiples; razón la cual emergen como un campo apropiado para generar una pluralidad de formas de conocimiento. Precisamente, en el ámbito de la investigación cualitativa coexisten profusas opciones de acercamiento a los fenómenos, el investigador puede operar comprensivamente con diversos paradigmas, sin pretender integrarlos. A lo anterior se puede adicionar el planteamiento formulado por Fernández y Postigo-Fuentes (2020), quienes establecen que:

Los investigadores interpretativos consideran a los individuos como capaces de construir su propia realidad social, criticando al positivismo en su concepción de que la realidad fuera objetiva y determinante en las percepciones y acciones del individuo: si la realidad es una construcción personal y social, esta determina la conducta en función de la interpretación, siendo por lo tanto en esta última donde debe enfocarse la investigación para comprenderla (pág. 48).

Ahora bien, aun cuando se reconoce la riqueza de posibilidades de acercamiento a los fenómenos, es prudente aceptar que para Vasilachis (2012d): "ningún método puede captar todas las sutiles variaciones de la experiencia humana" (pág. 13). Cada opción paradigmática presenta sistemas filosóficos particulares con su propia ontología, epistemología y metodología, 
elementos que en su conjunto demarcan las fronteras, aunque en algunos casos difusas, entre los diferentes modos de acercamiento al objeto de estudio.

Resulta claro que la valoración cualitativa de los fenómenos brinda la posibilidad de descubrir y redescubrir realidades, permitiéndole al investigador aportar interpretaciones y argumentaciones desde una pluralidad de posiciones. No obstante, el gran reto para el investigador cualitativo es vencer, señalando a Vasilachis (2012e): "el énfasis en la inferencia causal" que caracteriza las visiones positivistas (pág. 13); énfasis que trae como consecuencia directa la exclusión de formas alternas de apreciación y explicación de los fenómenos, provocando limitaciones en la anhelada comprensión profunda de los mismos.

Se debe agregar que, específicamente en el campo de las ciencias sociales, se hizo necesaria la valoración cualitativa de los fenómenos, dada la divergencia entre la teoría y la práctica, así como la ausencia de compromiso social que propendiera a la solución de problemas reales. De este modo, el conocimiento pasa a ser concebido como un fenómeno colectivo y socialmente construido, ocupando la óptica cualitativa el papel de puente entre la investigación como proceso formal y las necesidades de conocimiento de la sociedad; abriéndose paso a la investigación cogenerativa, la cual se sustenta en la colaboración y apunta a la búsqueda de soluciones plausibles a problemas de la vida real.

En adición a lo anterior, un elemento crucial en los abordajes cualitativos es el determinado por la relación del investigador con aquellos con quienes actúa. Al respecto, Álvarez-Gayou (2003b): sostiene que "los investigadores cualitativos son sensibles a los efectos que ellos mismos causan sobre las personas que son objeto de su estudio" (pág. 24); esto hace que emerja como necesario interrogarse sobre el papel de los actores, inquiriendo sobre la posibilidad de que sus aportes solo nutran las 
afirmaciones del investigador o si, por el contrario, se consideran sujetos dotados de voz, con propuestas y dilemas específicos, que mantienen lazos de reciprocidad con el investigador.

En atención a lo mencionado, se evidencia la necesidad de desarrollar formas de involucramiento más asertivas, procurando lograr un reposicionamiento discursivo de los investigadores sustentado en relaciones más cercanas y transparentes con los sujetos estudiados; esto implica sumergirse al interior de los discursos, pues en ellos se encuentran las aclaraciones y significados. Así, se rescata el valor de la otredad en el proceso de comprensión interpretativa de las vivencias y realidades humanas; aspecto que transfigura la relación entre el investigador y los actores participantes.

Por lo anterior, se infiere que el abordaje cualitativo enriquece las posibilidades del quehacer investigativo, al tiempo que impone nuevos y constantes desafíos al investigador, pues le interpela a reconocer la multiplicidad de formas de conocimiento y a profundizar sobre el alcance de tales formas, atendiendo a los sistemas filosóficos privativos de cada postura paradigmática que pueda asumir.

Una exigencia adicional que se impone al investigador cualitativo es el inexcusable desprendimiento de juicios de valor y de preconceptos acerca de los actores y sus realidades, para adentrarse en el campo con la mejor disposición de realizar interpretaciones que representen la multiplicidad de voces y sentidos que nacen de las experiencias inéditas de los actores. Reconociéndose que son estos últimos quienes determinan la construcción, validación y legitimación del conocimiento, como medio para hacer visibles sus sentires y vivencias particulares; así como de mostrar su alteridad como rasgo valioso e irreductible que los define.

Es conveniente poner de manifiesto la relevancia de la triangulación, en particular en la investigación en ciencias sociales, considerando que el esfuerzo del investigador por interpretar el mundo externo lo lleva a sumergirse 
en el imaginario multidiverso de los sujetos cuyos mundos se desentrañan, por lo que requiere herramientas que capten el polimorfismo de la realidad y estrategias que le lleven a certificar la veracidad de los hallazgos.

Justamente con el propósito de valorar la validez interna, que alude al grado en que las reconstrucciones interpretativas de la realidad son válidas, se ha utilizado la triangulación como una opción para acrecentar la robustez y calidad de un estudio cualitativo. Un primer acercamiento conceptual permite entenderla como la aplicación y combinación de varias metodologías de la investigación en el abordaje de un mismo objeto de estudio, hecho que la convierte en una herramienta que adiciona solidez, riqueza y complejidad a las investigaciones ante la imposibilidad de capturar la realidad objetiva.

De allí se colige que su aplicación propende a aumentar la comprensión de los fenómenos, para vencer el carácter inasible de la realidad objetiva, sustentándose en el empleo de diversos cristales que permiten reflejar externalidades y ayudan a refinar la agudeza interpretativa.

Más allá del intríngulis de las precisiones semánticas y conceptuales que emergen en torno al término, conviene examinar los aspectos pragmáticos inherentes. En este sentido, la triangulación admite el empleo de diferentes estrategias para estudiar el mismo problema, lo cual se expresa mediante la aplicación de diferentes técnicas para obtener los datos, la presencia de diferentes investigadores para un mismo análisis, la consideración de diversas fuentes de información o la asunción de diferentes teorías para explicar un mismo fenómeno; todo ello con la intención de reforzar el conocimiento y poder verificar la validez de los resultados.

En esta dirección se puede afirmar que la conjugación de múltiples estrategias para abordar el objeto en un estudio singular, expresadas en la aplicación coordinada de una diversidad de métodos, técnicas, perspectivas y observadores es una herramienta que adiciona rigor, amplitud y profundidad a cualquier investigación, ampliando el campo comprensivo; de esta forma la 
triangulación, como aplicación de diversas metodologías al análisis de una misma realidad social, le permite al investigador evaluar el grado en que son válidos los resultados dentro del contexto de la calidad metodológica del estudio.

Al trasladarnos al ámbito de la investigación educativa, la conceptualización se puede precisar; así es posible considerar lo declarado por Rodríguez, Pozo y Gutiérrez (2006), para quienes:

[...] La triangulación en el campo de la educación consiste en una estrategia de investigación mediante la cual un mismo objeto de estudio pedagógico es abordado desde diferentes perspectivas de contraste o momentos temporales donde la triangulación se pone en juego al comparar datos; contraponer las perspectivas de diferentes investigadores; o comparar teorías, contextos, instrumentos, agentes o métodos de forma diacrónica o sincrónica en el tiempo (pág. 289-290).

Se debe agregar además que, como forma de reforzar el conocimiento alcanzado sobre la realidad estudiada y poder verificar la validez y consistencia de los hallazgos, la triangulación conduce a encontrar referentes que señalen convergencia para poder efectuar una interpretación más densa, aunque no omnímoda, del fenómeno objeto de estudio; evitando de esta forma, el riesgo de sesgos y fallas metodológicas inherentes a las estrategias empleadas.

En este orden de ideas, es posible enunciar que la finalidad intrínseca de la triangulación es alcanzar una visión más completa del fenómeno en estudio, al fusionar diferentes perspectivas que intentan apreciar una única dimensión de la realidad. Tal herramienta conducirá a determinar intersecciones a partir de diferentes apreciaciones y fuentes informativas; convergencias que facilitan el proceso de corroboración estructural de la teoría creada o de la visión alcanzada de la realidad.

Es posible inferir que una ventaja destacable de la triangulación es la 
derivada del hecho de que, al utilizar diferentes métodos en la misma, éstos actúan como filtros que permiten decantar las singularidades de la realidad, lo que conlleva a obtener un mayor nivel de concreción y objetividad en los resultados analizados. Indiscutiblemente, las bondades de la triangulación superan su ubicación como un proceso de validación convergente, pues supone la creación de una visión holística del objeto de estudio, al ensanchar los límites de la comprensión de la realidad estudiada.

Es preciso añadir que la triangulación le permite al investigador superar la tendencia a aceptar de manera tácita la validez de sus impresiones iniciales; esto supone la minimización de los errores que surgen cuando el fenómeno es explorado desde la óptica de un solo observador, con la exclusividad de una técnica y desde un solo ángulo de observación. Implicando además tamizar tales impresiones iniciales para encontrar puntos de convergencia, 0 divergencia, entre las diferentes perspectivas desde las cuales se examina el objeto de estudio.

Así pues, la triangulación constituye una herramienta para disipar los sesgos derivados de las debilidades propias de los métodos empleados, de la adscripción teórica del investigador y de las interpretaciones erróneas que pueden presentarse a lo largo de la labor investigativa.

Ahora bien, dada la intencionalidad de encontrar intersecciones y convergencias en las apreciaciones que desde diferentes ángulos se hagan de la realidad, se tiende a aceptar que la similitud de los resultados aportados por diversas estrategias es prueba fehaciente de la certidumbre de los hallazgos; y que, por el contrario, la divergencia de los resultados brinda mediante la triangulación la oportunidad de conformar una perspectiva más dilatada para la interpretación del fenómeno abordado.

Conviene subrayar que una de las expectativas erróneas en torno a la triangulación es que mediante ésta se alcanzan de manera inequívoca resultados análogos al emplear diferentes estrategias, tal hecho, desestimado 
por su imposibilidad en un sentido estricto, tampoco es deseable, habida cuenta de que bajo la perspectiva cualitativa el conocimiento es una creación que se construye mediante la interacción entre el investigador y lo investigado, y por tanto ofrece la oportunidad de que existan múltiples versiones de la realidad indistintamente válidas.

Por tanto, al evaluar el fenómeno desde una perspectiva diferente se logran captar diversas facetas de la totalidad de la realidad en estudio, las cuales no necesariamente son coincidentes, haciendo esto que la triangulación sea una herramienta enriquecedora que procura captar la variedad multidimensional de los fenómenos y no un filtro de convergencias.

Así, entendiendo que la triangulación es un procedimiento heurístico encaminado a fundamentar y contrastar información desde diferentes ópticas es posible concebirla como una forma de validez que implica la proyección de apreciaciones múltiples de la realidad, cuyas tonalidades de luces y sombras se entremezclan a partir de los diferentes focos de contraste. Cabalmente, en el abanico de posibilidades que se abre al fusionar diferentes ópticas de acercamiento a la realidad se da paso a la metodología mixta, perspectiva desde la cual se pueden asumir profusas alternativas de integración sistemática de los métodos de investigación, según los propósitos del estudio y el matiz particular que le asigne el investigador atendiendo a criterios pragmáticos metodológicos. En atención a lo expuesto, emerge como oportuno presentar el planteamiento de Forni y De Grande (2020), quienes exponen que:

Revisamos un modo de concebir la investigación social que desde los márgenes de la oposición cualitativo-cuantitativo (o comprensivo-cualitativo) ha avanzado en la dirección de sostener que ambos paradigmas de producción de conocimiento no serían mutuamente excluyentes sino que, por el contrario, sería viable una articulación productiva entre ambos (pág. 160). 
De allí se infiere que la complementariedad metodológica se presenta como una vía fructífera para abordar las complejas realidades sociales, donde subyacen relaciones e interacciones que componen tejidos multidiversos que ameritan acercamientos multifocales a fin de aprehender en plenitud sus rasgos y esencias definitorias.

Por otra parte, retomando la centralidad de la triangulación como punto de atención, hay que mencionar además la propuesta planteada por Ruiz (2012a): relativa a la triangulación holística. El referido autor establece que ésta se genera a partir de la combinación de diversas estrategias de triangulación parcial, que se aplican durante las fases del proceso de la investigación.

Con la finalidad de precisar la conceptualización de triangulación parcial puede considerarse lo determinado por Alzás y Casa (2017): quienes la definen como "[...] aquella multiestrategia que se realiza en una sola fase del proceso de investigación o sobre uno de los elementos del proceso" (pág. 410). Esto indica que se desarrolla tal triangulación en procesos como la elección del paradigma, en el proceso de recolección de información y en la escogencia de las alternativas de análisis.

Cabe destacar, que la idea central en torno a la cual emerge el constructo triangulación se sustenta en la consideración de esta como estrategia de investigación, lo que se muestra acorde con la evolución del concepto en lo referente a su desarrollo teórico. Sin embargo, Ruiz (2012b): ofrece cierta amplitud en la concepción al considerarla como una técnica de control de la calidad de una investigación multiestratégica, sustentada en la viabilidad de "alternar planteamientos distintos para abordar un mismo problema, controlando así y elevando el nivel de calidad de sus conclusiones" (pág. 338); dejando ver el impacto de esta, más allá del diseño y desarrollo de la investigación, en la fase de elaboración de las conclusiones.

A partir de la perspectiva del referido autor, la triangulación también 
puede ser entendida como enriquecimiento, en virtud de que procura, a juzgar por Ruiz (2012c): "descubrir nuevos elementos de un objeto ya analizado, aumentar su estándar de precisión y corroborar su consistencia" (pág. 332). Por consiguiente, su concepción de triangulación se establece sobre tres pilares: control de la calidad de la investigación, la oportunidad de avalorar las conclusiones y reforzamiento de la validez, expresada esta última en términos de precisión y consistencia.

Focalizar la atención en las conclusiones tiene una significación particular en la investigación cualitativa, donde la construcción del conocimiento se da mediante un proceso subjetivo e intersubjetivo, pues ellas constituyen información esencial para validar epistemológicamente el proceder del investigador que sustenta su actuación en una racionalidad hermenéutica. Se colige entonces que el cruce dialéctico que se efectúa sobre la información pertinente al objeto de estudio soporta el corpus de resultados de la investigación, de allí el carácter primordial que asume la atención a la triangulación durante el proceso investigativo.

\section{Conclusiones}

Ser conscientes de la multidiversidad y complejidad de las realidades sociales permite al investigador expandir sus horizontes hacia objetos de estudio que se manifiestan crípticamente y solapan sus esencias detrás de las voces y sentimientos de los actores. Partiendo de la convicción de que el carácter no tangible de los hechos sociales remite a su obligada interpretación, se precisa la consideración de un cristal multifocal que conduzca al investigador a desplegar acercamientos diversos sobre su objeto de estudio; así, recurrir a esas visiones complementarias permite entrelazar las percepciones para generar una interpretación construida.

Para traducir los significados y dimensiones impalpables de las experiencias de terceros, respetando su alteridad, la investigación cualitativa 
emerge como la vía más expedita para adentrarse en las interioridades y plasmar el mundo interpretado, abandonando las certezas y las observaciones uniformes que implican los posicionamientos cuantitativos; constituyendo un campo emergente de saberes.

En ese intento por exteriorizar lo interior e interpretar las cosmovisiones, el investigador elabora una pintura cuyos matices permiten asignar significado y delinear, desde el prisma particular que se haya empleado, un esbozo de la realidad que no dejará de ser ajena, pero se devela rompiendo las fronteras invisibles que la separan de otros sentires y vivencias singulares. En este escenario, sistematizar resultados se carga de incertidumbre y demanda un alto grado de sensibilidad por parte del investigador que inquiere por realidades que le son ajenas y difusas; darle coherencia y confirmar la transparencia de los hallazgos se convierte en una tarea esencial y espinosa, más aún cuando las miradas circunspectas y agudas de terceros se afinan para encontrar debilidades.

De esta manera, la búsqueda incesante de criterios de calidad y rigor que otorguen robustez al proceso investigativo de orden cualitativo constituye una tarea insoslayable; comprender el carácter imperioso de esta demanda se facilita cuando se reconoce la notabilidad de valorar lo difuso y lo inédito. Lo descrito permite elucidar la esencialidad de la triangulación en la investigación cualitativa y reafirma su condición de herramienta enriquecedora que avalora el estudio al conferirle robustez, firmeza, agudeza y complejidad, concediéndole diversos niveles de solidez a los hallazgos y consolidando su fortaleza metodológica.

Esto ratifica su inexcusable aplicación en los estudios cualitativos, pues las realidades humanas son irreductibles a miradas parciales y fragmentadas; en este caso, la triangulación deja ver su bondad para aumentar la visión del panorama del fenómeno humano estudiado, procurando plasmar la esencia de este, desde diferentes prismas a partir de cruces dialécticos que nutren su 
comprensión por medio de miradas disimiles que lo hacen más inteligible.

\section{Referencias}

Alzás, T., \& Casa, L. (2017). La evolución del concepto de triangulación en la investigación social. Revista Pesquisa Qualitativa, 5(8), 395-418, eISSN 2525-8222. Recuperado de:

https://editora.sepq.org.br/rpq/article/view/95

Álvarez-Gayou, J. (2003a,b). Cómo hacer investigación cualitativa:

Fundamentos y Metodología. 1ra edición, ISBN: 968-853-516-8.

México, D.F.: Editorial Paidós Mexicana, S. A.

Delgado, Y., \& Alfonzo, R. (2019). Competencias Investigativas del Docente

Construidas durante la Formación Universitaria. Revista Scientific, 4(13), 200-220, e-ISSN: 2542-2987. Recuperado de:

https://doi.org/10.29394/Scientific.issn.2542-2987.2019.4.13.10.200-220

Denzin, N., \& Lincoln, Y. (2012a,b,c). La investigación cualitativa como disciplina y como práctica. En Denzin, N., \& Lincoln, Y. (coords.). El campo de la investigación cualitativa: Manual de investigación cualitativa. Vol. I. (págs. 43-102). ISBN 978-84-9784-308-9. Barcelona, España: Editorial Gedisa.

Fernández, M., \& Postigo-Fuentes, A. (2020). La situación de la investigación cualitativa en Educación: ¿Nueva Guerra de Paradigmas? Márgenes: Revista de Educación de la Universidad de Málaga, 1(1), 45-68, e-ISSN: 2695-2769. Recuperado de:

https://doi.org/10.24310/mgnmar.v1i1.7396

Flores, E., Meléndez, J., \& Mendoza, R. (2019). Producción científica como medio para la transformación social desde las universidades. Revista Scientific, 4(14), 62-84, e-ISSN: 2542-2987. Recuperado de: https://doi.org/10.29394/Scientific.issn.2542-2987.2019.4.14.3.62-84

Forni, P., \& De Grande, P. (2020). Triangulación y métodos mixtos en las 
Ensayo Original / Original Essay

ciencias sociales contemporáneas. Revista Mexicana de Sociología, 82(1), 159-189, e-ISSN: 0188-2503. Recuperado de:

http://revistamexicanadesociologia.unam.mx/index.php/rms/article/view $\underline{158064}$

Rodríguez, N. (2016). La formación del ciudadano desde la hermenéutica de lo ausente. RECIE: Revista Electrónica Científica de Investigación Educativa, 3(1), 59-64, e-ISSN: 2594-200X Recuperado de: https://www.rediech.org/ojs/2017/index.php/recie/article/view/185

Rodríguez, C., Pozo, T., \& Gutiérrez, J. (2006). La triangulación analítica como recurso para la validación de estudios de encuesta recurrentes e investigaciones de réplica en educación superior. RELIEVE, 12(2), 289-305, e-ISSN: 1134-4032. Recuperado de: https://doi.org/10.7203/relieve.12.2.4231

Ruiz, J. (2012a,b,c). Metodología de la investigación cualitativa. 5ta edición, ISBN: 978-84-9830-673-6. Serie Ciencias Sociales, vol. 15. Bilbao, España: Universidad de Deusto.

Tua, A. (2021). Actitud de los Maestrantes de Investigación Educacional ante la Elaboración del Trabajo de Grado. Revista Scientific, 6(19), 22-42, e-ISSN: 2542-2987. Recuperado de:

https://doi.org/10.29394/Scientific.issn.2542-2987.2021.6.19.1.22-42

Ugalde, N., \& Balbastre, F. (2013). Investigación cuantitativa e investigación cualitativa: buscando las ventajas de las diferentes metodologías de investigación. Ciencias Económicas, 31(2), 179187, e-ISSN: 0252-9521. Recuperado de:

https://revistas.ucr.ac.cr/index.php/economicas/article/view/12730

Varela, M., \& Vives, T. (2016). Autenticidad y calidad en la investigación educativa cualitativa: multivocalidad. Investigación en Educación Médica, 5(19), 191-198, e-ISSN: 2007-5057. Recuperado de:

http://dx.doi.org/10.1016/j.riem.2016.04.006 
Instituto Internacional de Investigación y Desarrollo Tecnológico Educativo INDTEC, C.A.

DOI: https://doi.org/10.29394/Scientific.issn.2542-2987.2021.6.20.15.275-295

OAI-PMH: http://www.indteca.com/ojs/index.php/Revista Scientific/oai

Ensayo Original / Original Essay

Vasilachis, I. (2012a,b,c,d,e). De las nuevas formas de conocer y de producir conocimiento. En Denzin, N., \& Lincoln, Y. (coords.). El campo de la investigación cualitativa: Manual de investigación cualitativa. Vol. I. (págs. 11-22). ISBN 978-84-9784-308-9. Barcelona, España: Editorial Gedisa. 


\section{Ensayo Original / Original Essay}

\section{Angela Esther Torres Ruiz}

e-mail: angtruiz@hotmail.com

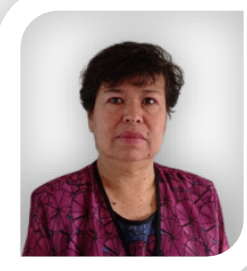

Nacida en San Cristóbal, Venezuela, el 23 de febrero del año 1966. Docente Titular de la Universidad Nacional Experimental del Táchira (UNET); Licenciada en Educación, Mención Matemática de la Universidad de Los Andes (ULA); Ingeniero de Sistemas de la Universidad Nacional Abierta (UNA); alcancé el título de Magister en Enseñanza de las Ciencias Básicas Mención Matemática de la Universidad Nacional Experimental del Táchira y Magíster en Matemática Mención Educación Matemática de la Universidad Nacional Experimental del Táchira; Doctora en Innovaciones Educativas de la Universidad Nacional Experimental Politécnica de la Fuerza Armada (UNEFA); Autora de los libros: Principios Básicos de Graficación y 745 Límites a tu alcance: una aproximación a su entendimiento.

El contenido de este manuscrito se difunde bajo una Licencia de Creative Commons ReconocimientoNoComercial-Compartirlgual 4.0 Internacional 